\title{
ASPECTOS ESTRUTURAIS DE ALGUMAS NEOLIGNANAS HIDROBENZOFURÂNICAS ${ }^{\#}$
}

\section{Massayoshi Yoshida}

Instituto de Química, Universidade de São Paulo, Av. Prof. Lineu Prestes, 748, 05508-900 São Paulo - SP / Centro de Biotecnologia da Amazônia, Av. Gov. Danilo de Matos Areosa, 690, 69075-351 Manaus - AM, Brasil

Recebido em 3/5/12; aceito em 2/7/12; publicado na web em 5/10/12

\begin{abstract}
STRUCTURAL ASPECTS OF SOME HYDROBENZOFURAN NEOLIGNANS. The neolignans are defined as dimers of allylphenol and propenylphenol between itself or crossed, whose bond does not occur by the $8-8$ ' carbons like lignans. This review centered on stereochemical aspects of the hydrobenzofuran type, a widespread skeleton among neolignans. The chemical structures established based on spectrometric data are registered in the literature. The absolute configurations reported previously were determined by chiroptical techniques. Some chemical transformations with neolignans, performed in previous studies, afforded products which are accumulated in other vegetal species and contributed to assign the unknown stereochemistry of these natural compounds. Possible biosynthetic pathways are also proposed.
\end{abstract}

Keywords: neolignans; hydrobenzofuran skeleton; Lauraceae.

\section{INTRODUÇÃO}

Em 1984, Gottlieb e Yoshida publicaram nesta revista uma revisão intitulada "Lignóides com Atenção Especial à Quimica das Neolignanas", ${ }^{1}$ introduzindo conceitos, nomenclatura, ocorrências, estruturas químicas, atividades biológicas, rearranjos com modificações de esqueleto carbônico, transformaçõos químicas e proposições biossintéticas.

O presente relato é uma coletânea sistematizada de trabalhos sobre neolignanas com esqueleto hidrobenzofurânico, abordando os aspectos estereoquímicos destas micromoléculas e a proposição biossintética destes compostos naturais.

As neolignanas, uma subclasse de lignoides, foram introduzidas na literatura química pelos trabalhos desenvolvidos no Laboratório de Química de Produtos Naturais do Instituto de Química da Universidade de São Paulo. Este Laboratório foi fundado e dirigido pelo Prof. O. R. Gottlieb com a colaboração inicial do Prof. Raimundo Braz Filho, seguida pela colaboração da Profa. Nidia F. Roque. Inúmeros colaboradores de outras instituições e estudantes de pós-graduação contribuíram para a realização e divulgação de trabalhos sobre as neolignanas, metabólitos isolados de extratos preparados de espécies arbóreas principalmente das famílias Lauraceae e Myristicaceae, sendo as primeiras dispersas na Mata Atlântica e na Floresta Amazônica, e, as segundas, preponderantemente distribuídas na Amazônia.

\section{NEOLIGNANAS HEXA-HIDROBENZOFURÂNICAS}

A análise conjunta dos dados espectrométricos de Ressonância Magnética Nuclear de ${ }^{1} \mathrm{H}\left(\mathrm{RMN}\right.$ de $\left.{ }^{1} \mathrm{H}\right)$ e de Massas (EM) da porosina, isolada de imbuia [Ocotea porosa (Lauraceae)], da burchelina, ${ }^{2}$ isolada de Aniba burchellii (Lauraceae) e da guianina, ${ }^{3}$ isolada de Aniba guianensis (Lauraceae) possibilitou a proposição da estrutura relativa 1 para a porosina. ${ }^{4}$ Verificou-se posteriormente com o isolamento de outras neolignanas, que os deslocamentos químicos observados no espectro de RMN de ${ }^{1} \mathrm{H}$ da porosina não eram inteiramente compatíveis com a estrutura 1 (Figura 1).

*e-mail: myoshida@iq.usp.br

\#Artigo em homenagem ao Prof. Otto R. Gottlieb (31/8/1920-19/6/2011)<smiles>C=CC[C@]12C=C(OC)C(=O)C=C1O[C@H](c1ccc3c(c1)OCO3)C2C</smiles>

burchelina<smiles></smiles><smiles>C=CC[C@]12C=C(OC)C(=O)CC1O[C@H](c1ccc(OC)c(OC)c1)C2C</smiles>

porosina (1)

Figura 1. Estruturas de burchelina, guianina e porosina (1)

Para a retificação da estrutura 1 sintetizaram-se dois cromóforos enônicos oxigenados em $\alpha(2)$ e $\beta$ (3) à carbonila e registraram-se seus espectros na região do UV. A cada espectro do cromóforo adicionaram-se as absorbâncias encontradas no espectro no UV do veratrol. Observou-se que o espectro no UV resultante da adição dos espectros de $\mathbf{3}$ e veratrol foi mais coincidente com o espectro da porosina do que o espectro resultante da adição dos espectros de $\mathbf{2}$ e veratrol (Figura 2). ${ }^{5}$
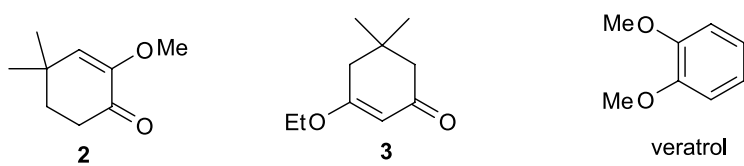

Figura 2. Estruturas de modelos dos cromóforos da porosina

A evidência conclusiva de que a metoxila alifática está localizada em C5' foi obtida pela eliminação da metoxila por fotólise, uma reação que ocorre somente em metoxilas ligadas a carbonos saturados. ${ }^{6}$ Ainda, o produto desmetoxilado em 5' apresentou o mesmo espectro no UV da porosina. Com base nessas evidências, a estrutura da porosina foi retificada de $\mathbf{1}$ para 4 (Figura 3).

A revisão da estrutura da porosina requereu uma reanálise da estrutura 5 da canelina-B, isolada de Licaria canella (Lauraceae), ${ }^{7}$ pois a sua proposição estrutural havia sido baseada na estrutura 1 . As 
<smiles>C=CC[C@]12C=C(OC)C(=O)CC1OC(c1ccc(OC)c(OC)c1)C2C</smiles><smiles>C=CCC12CC(OC)C(=O)C=C1OC(c1ccc(OC)c(OC)c1)C(C)C2C</smiles>

Figura 3. Revisão de estrutura da porosina

evidências para a retificação da estrutura de $\mathbf{5}$ para $\mathbf{6}$ foram obtidas através da técnica de deslocamento induzido de hidrogênios no espectro de RMN de ${ }^{1} \mathrm{H}$ pelo reagente tris-(6,6,7,7,8,8,8-heptafluor-2,2-dimetil-3,5-octanodionato) de Praseodímio [Pr(FOD) 3 ], Figura 4. O deslocamento induzido $\Delta \delta$ das metoxilas da canelina-B mostrou que uma das metoxilas é coplanar à carbonila $(\Delta \delta 27,0)$ e a outra situa-se fora do plano da carbonila $(\Delta \delta 6,5)$ e, ainda, para corroborar a evidência, o $\Delta \delta$ do hidrogênio carbinólico $5^{\prime}$ 'da porosina $(\Delta \delta 25,0)$ é semelhante ao hidrogênio carbinólico $5^{\prime}$ da canelina-B $(\Delta \delta 21,0){ }^{8}$<smiles>C=CC[C@]12C(OC)=C(OC)C(=O)CC1OC(c1ccc3c(c1)OCO3)[C@@H]2C</smiles><smiles>C=CC[C@]12C[C@H](OC)C(=O)C(OC)=C1O[C@H](c1ccc3c(c1)OCO3)[C@H]2C</smiles>

Figura 4. Revisão de estrutura da canelina-B

Neolignanas hexa-hidrobenzofurânicas com diferenças configuracionais foram isoladas de Licaria armeniaca (Lauraceae) e as estruturas relativas estabelecidas como sendo 7 e 8 (Figura 5) designadas, respectivamente, de armenina-A e armenina-B. ${ }^{9}$<smiles>C=CC[C@]1(C)CC(OC)C(=O)C=C1O[C@H](c1ccc2c(c1)OCO2)C(C)C</smiles><smiles>C=CC[C@]12CC(OC)C(=O)C(OC)=C1O[C@H](c1ccc3c(c1)OCO3)C2C</smiles>

Figura 5. Estruturas de armenina-A e armenina-B

Mais tarde, o número de neolignanas hexa-hidrobenzofurânicas foi acrescido com o isolamento de compostos com estruturas $\mathbf{9 , 1 0}$ e 11 (Figura 6), dos extratos de Aniba sp, (Lauraceae), designadas de canelina-D, canelina-E e armenina-C, respectivamente. ${ }^{10}$<smiles>C=CC[C@]12CC(=O)C(OC)C=C1O[C@H](c1cc(OC)c(OC)c(OC)c1)[C@H]2C</smiles><smiles>C=CC[C@]12CC(OC)C(=O)C(OC)=C1OC(c1cc(OC)c3c(c1)OCO3)[C@@H]2C</smiles><smiles>C=CC[C@]12CC(OC)C(=O)C=C1O[C@H](c1cc(OC)c3c(c1)OCO3)C(C)C2C(C)(C)C</smiles>

Figura 6. Estruturas de canelina-D, canelina-E e armenina-C
Ainda, uma análise dos extratos da madeira do tronco de Ocotea porosa coletado no Instituto Botânico (São Paulo - SP) forneceu entre outras, duas neolignanas hexa-hidrobenzofurânicas com estruturas $\mathbf{1 2}$ e 13 (Figura 7), que foram designadas, respectivamente, de porosina-C e canelina-F. ${ }^{11}$<smiles>C=CCC12C[C@@H](OC)C(=O)C=C1OC(c1ccc3c(c1)OCO3)C2C</smiles><smiles>C=CC[C@]12CC(OC)C(=O)C=C1OC(c1ccc3c(c1)OCO3)[C@H](C)[C@@H]2C(F)(F)F</smiles>

Figura 7. Estruturas de porosina-C e canelina- $F$

As neolignanas hexa-hidrobenzofurânicas isoladas foram agrupadas em três tipos estruturais e foram designadas de porosinas (A, $\mathrm{B}$ e C), canelinas (B, D, E e F) e armeninas (A, B e C). As porosinas apresentam os grupos arílico e metílico em cis e a metila absorve em ca. $0,5 \delta$ no espectro de $\mathrm{RMN}$ de ${ }^{1} \mathrm{H}$. As canelinas e as armeninas possuem grupos arílico e metílico em trans e as metilas absorvem em RMN de ${ }^{1} \mathrm{H}$ entre 1,0 a 1,2 $\delta$. A relação cis dos grupos metílico e alílico nas canelinas e a relação destes grupos em trans nas armeninas mostram diferenças na absorção da metila no espectro de RMN de ${ }^{13} \mathrm{C}$ devido ao efeito $\gamma$ de proteção diamagnética. Assim, na relação cis, C9 da canelina absorve em 11,7 $\delta$ e na relação trans da armenina C9 absorve em 17,5 $\delta$ nos respectivos de espectros de RMN de ${ }^{13} \mathrm{C}$.

\section{DETERMINAÇÃO DA CONFIGURAÇÃO ABSOLUTA}

A estereoquímica absoluta dos grupos metílico e arílico no anel furânico de di-hidrobenzofuranos havia sido estabelecida e descrita na literatura para melanoxina ${ }^{12}$ e para obtusafurano. ${ }^{13}$ A melanoxina e o obtusafurano (Figura 8) serviram de modelos para a configuração absoluta de neolignanas.<smiles>COc1ccc([C@@H]2Oc3cc(OC)c(O)cc3[C@@H]2C)cc1O</smiles><smiles>COc1cc2c(cc1O)[C@H](C)[C@H](c1ccccc1)O2</smiles>

Figura 8. Estruturas de melanoxina e obtusafurano

A burchelina foi submetida à aromatização do anel dienônico na presença de $\mathrm{PtO}_{2}$ fornecendo um produto de estrutura 14 (Figura 9). ${ }^{2}$ A configuração relativa trans dos grupos metílico e arílico de $\mathbf{1 4}$ foi estabelecida comparando-se o deslocamento químico de hidrogênios da metila em C9 com os deslocamentos químicos dos modelos melanoxina e obtusafurano. A curva de dispersão rotatória óptica (DRO) de $\mathbf{1 4}$ mostrou-se idêntica à curva de DRO da melanoxina ${ }^{14}$ e de sinal contrário à curva de DRO da obtusafurano. ${ }^{15}$ Atribuiu-se, assim, a configuração $7 S, 8 S$ para 14 e esta configuração estende-se também aos centros estereogênicos da burchelina.<smiles>C=CCC12C=C(OC)C(=O)C=C1O[C@H](c1ccc3c(c1)OCO3)C(C)C2C</smiles><smiles>COc1cc2c(cc1O)OC(c1ccc3c(c1)OCO3)[C@H]2C</smiles>

Figura 9. Estrutura do derivado 14 da burchelina

Para porosina, havia a necessidade de conhecer as propriedades ópticas da luz polarizada do grupo ciclo-hexenônico. A porosina 
foi oxidada pelo diciano-diclorobenzoquinona (DDQ) obtendo-se o produto 15, eliminando-se a dissimetria de C7 e C8 (Figura 10). A curva de dicroísmo circular (DC) de $\mathbf{1 5}$ mostrou-se idêntica às curvas de DC da porosina-A, canelina-D e armenina-A na região de absorção do cromóforo enônico em $\lambda c a .305 \mathrm{~nm}$. Pôde-se assim concluir que as porosinas, canelinas e armeninas possuíam a mesma configuração absoluta $l^{\prime} R .^{10}$<smiles>C=CCC12CC(OC)C(=O)C=C1OC(c1ccc(OC)c(OC)c1)C2C</smiles><smiles>C=CCC12CC(OC)C(=O)C=C1OC(c1ccc(OC)c(OC)c1)=C2C</smiles>

Figura 10. Estrutura do derivado 15 da porosina

A comprovação da estereoquímica foi reforçada com base nos dados espectrométricos obtidos para as mirandinas A e B, isoladas de Nectandra miranda (Lauraceae), e a epi-mirandina-A, obtida pela isomerização da mirandina-A em meio ácido. ${ }^{16}$ As mirandinas diferem da burchelina por apresentarem um grupo metoxílico em lugar de um grupo alílico em C5'. As mirandinas A e B apresentam relação trans entre os grupos metílico e arílico e os hidrogênios metílicos absorvem no espectro de $\mathrm{RMN}$ de ${ }^{1} \mathrm{H}$, respectivamente, em $1,11 \delta$ e $1,15 \delta$. A diferença na configuração do grupo metoxílico causa distinções nos deslocamentos químicos de RMN de ${ }^{1} \mathrm{H}$ de seguintes grupos, respectivamente, para mirandina A e mirandina $\mathrm{B}$ : MeO-5' $3,00 \delta$ vs $3,12 \delta$; H-7 5,16 $\delta$ vs $5,32 \delta$; H- $82,65 \delta$ vs $2,16 \delta$. Os hidrogênios metílicos em C9 da epi-mirandina-A absorvem no espectro de $\mathrm{RMN}$ de ${ }^{1} \mathrm{H}$ em 0,52 $\delta$ e o H-7 em 6,06 $\delta$, indicando uma relação cis entre os grupos metílico e arílico. O produto aromatizado $\mathbf{1 6}$ no anel dienônico da mirandina-A mostrou curva de DRO idêntica à curva de DRO da melanoxina, permitindo atribuir as configurações $7 S, 8 R$ para a mirandina-A e, consequentemente, a configuração 5 'S para mirandina-A e $5^{\prime} R$ para a mirandina-B (Figura 11 ).<smiles>C=CCC1=CC(=O)C(OC(c2cc(OC)c(OC)c(OC)c2)C(C)C)CC1C</smiles><smiles>C=CCC1=C[C@]2(O)C(=CC1=O)O[C@H](c1cc(OC)c(OC)c(OC)c1)[C@H](C)[C@H]2C</smiles><smiles>C=CCc1cc2c(cc1O)O[C@H](c1cc(OC)c(OC)c(OC)c1)C2C</smiles><smiles>C=CCC1=CC2(C)C(=CC1=O)OC(c1cc(OC)c(OC)c(OC)c1)C(C)C2OC</smiles>

epi-mirandina-A

Figura 11. Estruturas de mirandinas e derivados

\section{PRECURSORES DA POROSINA E SUAS REAÇÕES}

O fracionamento do extrato da madeira do tronco de imbuia, coletado na reserva do Instituto Botânico em São Paulo-SP, forneceu vários compostos anteriormente não isolados. Dois destes compostos, entre outros, tiveram as suas estruturas determinadas como sendo 17 e 18 (Figura 12) com base nos experimentos descritos a seguir

Os compostos 17 e $\mathbf{1 8}$ submetidos à oxidação por reagente de Jones forneceram um mesmo composto: a porosina. A reação de acetilação de $\mathbf{1 7}$ resultou em um monoacetato $\mathbf{1 9}$, com a acetoxila em equatorial. O composto $\mathbf{1 8}$, que possui uma hidroxila no $\mathrm{C} 4$ ' em axial, forneceu 20, por eliminação de $\mathrm{H}_{2} \mathrm{O}$, na presença de anidrido<smiles>C=CC[C@]1(C)C[C@H](OC)[C@@H](O)[C@H](O)[C@]1(O)OC(C)c1ccc(OC)c(OC)c1</smiles><smiles>C=CC[C@]12CC(OC)C(O)CC1(O)OC(c1ccc(OC)c(OC)c1)C2C</smiles><smiles>C=CC[C@]12C[C@H](OC)[C@@H](OC(C)=O)[C@H](O)[C@]1(O)O[C@H](c1ccc(OC)c(OC)c1)C2C</smiles><smiles>C=CCC1(C(O)C(C)c2ccc(OC)c(OC)c2)CC(OC)C=CC1=O</smiles><smiles>C=CCC1(C(C)C(O)c2cc(OC)c(OC)c(OC)c2)CC(OC)C=CC1=O</smiles>

Figura 12. Estruturas de precursores 17 e 18 da porosina, derivados $19 e$ 20 e megafona

acético e piridina. Os dados espectroscópicos de $\mathbf{2 0}$ são semelhantes aos dados descritos para a megafona isolada de Aniba megaphylla (Lauraceae). ${ }^{17}$ Como a estrutura absoluta da megafona havia sido estabelecida pelos raios- $\mathrm{X}$, as estruturas definidas para a porosina $\mathrm{e}$ outras neolignanas hexa-hidrobenzofurânicas tiveram suas estereoquímicas reconfirmadas.

Outra transformação notavel se refere à conversão da porosina-B isolada de Urbanodendrom verrucosum em um derivado $\mathbf{2 1},{ }^{18}$ análogo à futoenona, que havia sido isolada de Piper futokadzura (Piperaceae). ${ }^{19}$ Esta reação de conversão permite estabelecer a estereoquimica absoluta da complexa estrutura da futoenona (Figura 13).<smiles>C=CC[C@]12CC(OC)C(=O)C=C1OC(c1ccc3c(c1)OCO3)C2C</smiles><smiles>COC1C[C@]23CC(C[C@H](c4ccc5c(c4)OCO5)C2C)OC3=CC1=O</smiles>

21<smiles></smiles>

futoenona

Figura 13. Estruturas de porosina-B, derivado 21 e futoenona

\section{PROPOSIÇÕES BIOSSINTÉTICAS}

Segundo Gottlieb, as neolignanas eram definidas como dímeros de alilfenol e propenilfenol entre si ou cruzado. Atualmente, a IUPAC conceitua como neolignanas os dímeros de unidades $\mathrm{C}_{6}-\mathrm{C}_{3}$, que não estejam ligados através de ligações $8,8^{\prime}$ da cadeia alifática.

As lancifolinas isoladas de Aniba lancifolia (Lauraceae) apresentam-se como dímeros cuja etapa de ciclização para a formação do anel hidrobenzofurânico não ocorreu (Figura 14). ${ }^{20} \mathrm{O}$ isolamento dos isômeros lancifolinas A e B indica falta de seletividade na etapa 
de dimerização (Figura 15), reforçando o argumento de um possível envolvimento do tipo mecanismo radicalar como inicialmente proposto. A informação nova que pode ser deduzida é de que a unidade alilfenol requer uma hidroxila em 2 , fato que facilita a racionalização da proposição biossintética na formação do anel dienônico das lancifolinas e das mirandinas. A proposição biossintética de uma unidade alilfenol hidroxilado na posição 2 pode ser estendida a todas as neolignanas hidrobenzofurânicas.
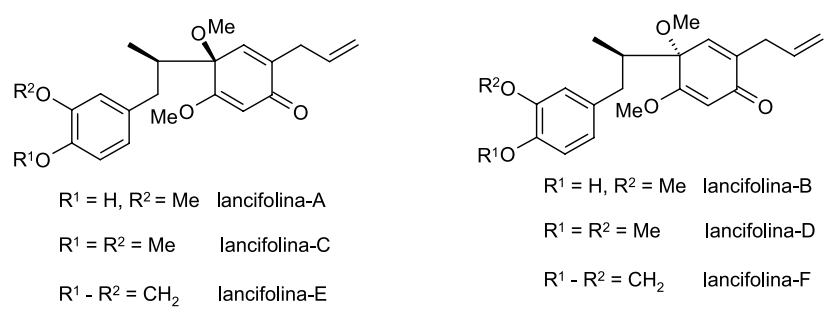

Figura 14. Estruturas de lancifolinas<smiles>C=CCC1=CC(OC)(C(C)C(C)Cc2ccc(O)c(OC)c2)C(OC)=CC1=O</smiles>

Figura 15. Biogênese de lancifolina

As mirandinas isoladas de Nectandra miranda apresentam a mesma falta de seletividade na dimerização, mas a ciclização do anel di-hidrofurânico é seletiva, existindo só uma forma configuracional do carbono oxibenzílico. ${ }^{16}$

A determinação da estereoquímica absoluta envolveu a comparação das curvas de DC do produto 22 de reação de aromatização do anel dienônico da lancifolina-F na presença de $\mathrm{Zn}$ e ácido acético e do produto $\mathbf{2 3}$ de hidrogenólise da licarina-B, isolada de Licaria aritu (Lauraceae), Figura $16 .{ }^{21} \mathrm{~A}$ interpretação das curvas de DC dos produtos 22 e $\mathbf{2 3}$ permitiu concluir que estes derivados possuem a mesma configuração $8 R$.<smiles>C=CCC1=CC(OC)(C(C)Cc2ccc3c(c2)OCO3)C(OC)=CC1=O</smiles><smiles>C=CCc1cc(C(C)Cc2ccc3c(c2)OCO3)c(OC)cc1O</smiles><smiles>C/C=C/c1cc(OC)c2c(c1)C(C)[C@H](c1ccc3c(c1)OCO3)O2</smiles><smiles>CCCc1cc(CC(C)C)c(O)c(OC)c1</smiles>
23
As neolignanas hidrobenzofurânicas são moléculas simples e pequenas, mas a diversidade configuracional de suas estruturas constitui um desafio e um estímulo para esclarecer e compreender a complexidade das moléculas na natureza.

\section{REFERÊNCIAS}

1. Gottlieb, O. R.; Yoshida, M.; Quim. Nova 1984, 7, 250.

2. Lima, O. A.; Gottlieb, O. R.; Magalhães, M. T.; Phytochemistry 1972, 11,2031 .

3. von Bulow, M. V.; Franca, N. C.; Gottlieb, O. R.; Puentes-Suarez, A. M.; Phytochemistry 1973, 12, 1805.

4. Aiba, C. J.; Braz-Filho, R.; Gottlieb, O. R.; Phytochemistry 1973, 12, 413.

5. Aiba, C. J.; Gottlieb, O. R.; Yoshida, M.; Mourão, J. C.; Gottlieb, H. E.; Phytochemistry 1976, 15, 1031.

6. Yoshida, M.; Weiss, R. G.; Tetrahedron 1975, 31, 1801.

7. Giesbrecht, A. M.; Franca, N. F.; Gottlieb, O. R.; Rocha, A. F. I.; Phytochemistry 1974, 13, 2285.

8. Cavalcante, S. de H.; Giesbrecht, A. M.; Gottlieb, O. R.; Mourão, J. C.; Yoshida, M.; Phytochemistry 1978, 17, 983.

9. Aiba, C. J.; Gottlieb, O. R.; Maia, J. G. S.; Pagliosa, F. M.; Yoshida, M.; Phytochemistry 1978, 17, 2038.

10. Trevisan, L. M. V.; Yoshida, M.; Gottlieb, O. R.; Phytochemistry 1984, 23,661 .

11. Dias, D. A.; Yoshida, M.; Gottlieb, O. R.; Phytochemistry 1986, 25, 2613.

12. Donnelly, B. J.; Donnelly, D. M. X.; O’Sullivan, A. M.; Prendergast, J. P.; Tetrahedron 1969, 25, 4409.

13. Gregson, M.; Ollis, W. D.; Redman, B. T.; Sutherland, I. O.; Chem. Commun. 1968, 1394.

14. Donnelly, D. M. X.; comunicação pessoal.

15. Redman, B. T.; PhD. Thesis, University of Sheffield, U. K., 1968, p. 96.

16. Aiba, C. J.; Gottlieb, O. R.; Pagliosa, F. M.; Yoshida, M.; Magalhães, M. T.; Phytochemistry 1977, 16, 745.

17. Kupchan, S. M.; Stevens, K. L.; Rohlfing, E. A.; Sickles, B. R.; Sneden, A. T.; Miller, R. N.; Bryan, R. F.; J. Org. Chem. 1978, 43, 586.

18. Dias, A. F.; Giesbrecht, A. M.; Gottlieb, O. R.; Phytochemistry 1982, 21, 1137.

19. Ogiso, A.; Kurabayashi, M.; Takahashi, S.; Mishima, H.; Woods, M. C.; Chem. Pharm. Bull. 1970, 18, 105.

20. Diaz-Diaz, P. P.; Yoshida, M.; Gottlieb, O. R.; Phytochemistry 1980, 19 , 285.

21. Aiba, C. J.; Campos-Correa, R. G.; Gottlieb, O. R.; Phytochemistry $\mathbf{1 9 7 3}, 12,1163$.

Figura 16. Estruturas de derivados 22 da lancifolina- $F$ e 23 da licarina- $B$ 